\title{
Terapia Dialéctica Conductual: aplicaciones clínicas y evidencia empírica
}

\author{
Joaquim Soler ${ }^{1,2}$, Matilde Elices ${ }^{1,2,3}$, Cristina Carmona ${ }^{1}$ \\ ${ }^{1}$ Servicio de Psiquiatría. Hospital de la Santa Creu i Sant Pau, Barcelona, España. \\ ${ }^{2}$ Centro de Investigación Biomédica en Red de Salud Mental (CIBERSAM). \\ Institut d'Investigació Biomèdica - Sant Pau (IIB-Sant Pau). \\ ${ }^{3}$ Programa de Cognición. Facultad de Psicología. Universidad de la República, Uruguay.
}

RESUMEN

La Terapia Dialéctica Conductual (TDC) es un tratamiento desarrollado para pacientes con Trastorno Límite de la Personalidad (TLP). En la TDC se combinan técnicas de terapia de la conducta con principios de aceptación de la realidad derivados del Zen y de la filosofía dialéctica. La TDC es una terapia multi-modal que incluye 4 componentes: terapia individual, entrenamiento grupal en habilidades, soporte telefónico y un grupo de consulta para los terapeutas. Como lo demuestran distintos ensayos clínicos controlados y aleatorizados, la TDC es hoy en día el único tratamiento para el TLP que ha sido suficientemente investigado como para considerarse basado en la evidencia. La TDC también ha sido adaptada para el tratamiento de otras patologías vinculadas a la disregulación emocional, lo que ha contribuido a ampliar su aplicación a otras poblaciones clínicas. En este sentido, el entrenamiento en habilidades también se aplica como componente único de tratamiento, acumulando cada vez más evidencia en relación a su eficacia. En el presente artículo se exponen los principios básicos de la terapia y se realiza una revisión de sus aplicaciones clínicas y así como de la evidencia empírica disponible hasta el momento.

Palabras clave: Terapia Dialéctica Conductual; evidencia empírica; aplicaciones.

\begin{abstract}
Dialectical Behavior Therapy (DBT) is a treatment developed for patients with borderline personality disorder diagnosis (BPD). DBT combines behavior therapy with reality acceptance principles derived from Zen and dialectical philosophy. DBT is a multi-modal treatment with 4 components: individual therapy, skills training, phone consultation and consultation team for therapists. Today, there are several randomized controlled clinical trials proving enough evidence as to consider that DBT is an empirically based treatment for BPD. DBT has also been adapted for the treatment of other clinical populations characterized by emotional dysregulation. The skills training component of the therapy has begin to be used as a standalone treatmentent and evidence in regard to its efficacy is still growing. In this article we resume the principles and structure of DBT and review its clinical applications and empirical data.
\end{abstract}

Keywords: Dialectical Behavior Therapy; empirical evidence; applications. 


\section{Principios y estructura}

\section{de la terapia dialéctica conductual}

La terapia dialéctica conductual (TDC; Linehan, 1993a) es un tratamiento desarrollado por Marsha M. Linehan para pacientes con múltiples conductas suicidas y diagnóstico de trastorno límite de la personalidad (TLP). El TLP es un trastorno severo, con una tasa de mortalidad del 8-10\% y caracterizado por una gran disregulación emocional, frecuentemente acompañada de una alta impulsividad y problemas en las relaciones interpersonales (American Psychiatric Association, 2013). Consecuentemente el TLP conlleva una elevada incapacidad funcional y se asocia a elevados costes para los servicios sanitarios.

Aunque existen actualmente varias formas de psicoterapia que se han mostrado eficaces para el tratamiento del TLP, es la TDC, con 11 estudios controlados y más de 570 pacientes aleatorizados en estos, la terapia que cuenta con mayor evidencia empírica hasta la fecha (Stoffers et al., 2012). En este sentido, y según la última revisión de la Cochrane, se puede afirmar que existe evidencia empírica suficiente como para considerar que la TDC en comparación con tratamiento habitual es eficaz para reducir la rabia, el comportamiento suicida, las conductas parasuicidas, la psicopatología asociada al trastorno, la ansiedad y aumentar la salud mental en general (Stoffers et al., 2012).

El aspecto más definitorio de la terapia de Linehan es la combinación de la terapia cognitivo conductual (TCC) clásica -focalizada en el cambio conductual- con principios que provienen del Zen - focalizados en la aceptación y la validación-. En este sentido la TDC es pionera en introducir dentro de la psicoterapia científica algunos procedimientos y técnicas actualmente muy utilizadas, como son el mindfulness o las estrategias de aceptación (Linehan, 1993a). Ante la experiencia frecuente de fracasos en la aplicación de TCC con pacientes con TLP, Linehan encuentra necesaria la integración de tecnologías de cambio (i.e. terapia conductual) y tecnologías de aceptación (i.e., Zen; Linehan, 1993a). Considerando la complejidad y la multiplicidad de problemas que presenta el paciente, se requiere una terapia que ponga énfasis en la solución de problemas. Pero cuando la terapia se focaliza únicamente en el cambio, los pacientes se sienten invalidados y con frecuencia abandonan el tratamiento. El extremo opuesto, o sea una terapia basada únicamente en la aceptación, tampoco resultaría efectiva al no promoverse los cambios necesarios para mejorar la situación del paciente. La TDC pretende entonces proveer un marco que permita lograr una síntesis entre el cambio y la aceptación.

La TDC no solo plantea un modelo de tratamiento, sino que además expone un modelo teórico en el que se basan sus principios terapéuticos. Según la teoría biosocial, el TLP es un trastorno caracterizado por una disfunción general del sistema de regulación emocional (Crowell, Beauchaine, \& Linehan, 2009; Linehan, 1993a). Las conductas desadaptativas que presenta el paciente (e.g., consumo de tóxicos, autolesiones, disregulación interpersonal) son consecuencia natural de esta falta de habilidades para regular las emociones (Linehan, 1993b). El modelo contempla factores de vulnerabilidad biológica y ambientales en la génesis de esta disregulación emocional que 
se relacionarían de forma transaccional ( Linehan, 1993a).

Para organizar el tratamiento se establecen unas prioridades basadas en el nivel de severidad de la problemática del paciente. De esta forma, se establece una jerarquía que guía al terapeuta para saber "qué" tratar y "cuándo" tratarlo. A su vez la terapia se estructura entorno a cuatro modos de intervención: (1) terapia individual, (2) entrenamiento en habilidades, (3) Ilamadas telefónicas, y (4) grupo de consulta para terapeutas (Linehan, 1993a), lo que actualmente se conoce como el formato estándar de TDC.

\section{Eficacia de la terapia dialéctica conductual para el tratamiento del trastorno límite de la personalidad}

En 1991, Linehan publica el primer ensayo clínico (Linehan, Armstrong, Suarez, Allmon, \& Heard, 1991), en el que 44 pacientes con diagnóstico de TLP y conductas parasuicidas recientes, fueron aleatorizadas a un año de TDC o un año de tratamiento habitual (TH). Las pacientes en el grupo de TDC mostraron una mayor disminución en frecuencia y severidad de las conductas parasuicidas, y en la frecuencia y duración de los días de ingreso, así como una mejor tasa de retención respecto al TH. Estas mejorías se mantuvieron a los 6 y 12 meses de seguimiento, observándose también una mejoría significativa en la Impresión Clínica Global (ICG), una disminución de la ira rasgo, y una mayor adaptación social (Linehan, Tutek, Heard, \& Armstrong, 1994). Ambos tratamientos fueron eficaces para disminuir la depresión, desesperanza, ideación suicida y aumentar las razones para vivir. A partir de este primer estudio, la evidencia en relación a la eficacia de la TDC ha ido creciendo de forma exponencial y así lo demuestra el creciente número de citas acumuladas desde el año 2000 hasta hoy. En la actualidad, hay un total de 11 ensayos randomizados y controlados llevados a cabo en sitios independientes en los que se compara la TDC versus el TH (Carter, Willcox, Lewin, Conrad, \& Bendit, 2010; Koons et al., 2001; Linehan et al., 1991; Linehan, Schmidt, Dimeff, \& Comtois, 1999; Linehan et al., 2002; Linehan et al., 2006; McMain et al., 2009; Pasieczny \& Connor, 2011; Soler et al., 2009; Turner, 2000; Verheul et al., 2003). En general, estos estudios reportan efectos beneficiosos en distintos aspectos de la sintomatología límite: conducta suicida, depresión y ansiedad (tamaños del efecto muy importantes), rabia (tamaño del efecto importante), conductas parasuicidas (tamaño del efecto moderado), y salud mental general (tamaño del efecto de moderado a importante). En aquellos estudios en los que la TDC fue evaluada en contraste con una rama de tratamiento más rigurosa que el TH, como por ejemplo el tratamiento según guías APA (McMain et al., 2009) o la terapia de expertos (Linehan et al., 2006), la TDC mostró superioridad en uno de los estudios (Linehan et al., 2006) pero no en el otro (McMain et al., 2009), siendo en este último ambas ramas de tratamiento eficaces en la mejoría de la clínica (i.e. conductas parasuicidas, suicidas y depresión).

\section{Otras aplicaciones clínicas}

Desde el primer estudio publicado a principios de los años 90, la mayor parte de la investi- 
gación en TDC se concentró en el estudio de la eficacia de la terapia para pacientes con diagnóstico de TLP y un alto grado de severidad y descontrol conductual. Los primeros estudios se focalizaron entonces en demostrar la eficacia de la TDC en estos aspectos, pero más tarde el interés comenzó a situarse en relación a la aplicabilidad de la TDC en otros trastornos o problemas clínicos. En un análisis post-hoc del estudio en el que se compara la TDC con tratamiento de expertos (Linehan et al., 2006), se estudia la eficacia de la TDC para el tratamiento de distintos trastornos del eje I comorbidos al TLP (Harned et al., 2008). Los resultados indican que la TDC es eficaz en la reducción de: trastorno por abuso de sustancias (reducción del 87\%), trastorno depresivo mayor (reducción del $68 \%$ ), trastornos de la alimentación (reducción del 64\%). Sin embargo, parecería ser que la TDC no es tan eficaz para reducir trastornos de ansiedad, como el trastorno por pánico (reducción del 47\%) o el trastorno por estrés postraumático (reducción del 35\%). Estos resultados indican la necesidad de mejorar la eficacia de la terapia en estos aspectos y por tanto, abren una línea de investigación.

En los últimos años, la aplicación de la TDC al segundo estadio de tratamiento (i.e., síntomas de estrés postraumático) ha dado lugar a varios estudios. Bohus y colaboradores publican en el año 2013 un estudio en el que se evalúa la eficacia de una adaptación de la TDC para individuos con TLP y diagnóstico de PTSD comórbido, víctimas de abuso sexual en la infancia (Bohus et al., 2013). Este programa combina el enfoque de la TDC con enfoques cognitivo-conductuales diseñados para el trauma y tiene 3 objetivos principales: (1) que el paciente reduzca el miedo a las emociones primarias relacionadas con el trauma, (2) reducir las emociones secundarias asociadas al trauma, (3) promover la aceptación radical del evento traumático. Un total de 74 pacientes (de las cuales aproximadamente el 50\% cumplía con criterios para el TLP) fueron randomizados a 12 semanas de DBT-PTSD (tratamiento intensivo en unidades de hospitalización) o tratamiento usual (lista de espera). Pacientes que se autolesionaban en el momento del estudio fueron excluidos. Los resultados muestran una reducción significativa en relación a los síntomas de PTSD (Bohus et al., 2013). Por otra parte, el equipo de Linehan también desarrolló un protocolo para pacientes con TLP y PTSD, incluyendo en sus estudios (a diferencia de Bohus), pacientes que presentan autolesiones. Este protocolo consiste en TDC estándar más un programa de exposición prolongada (EP; Foa, Riggs, Massie, \& Yarczower, 1995). Tanto en el estudio piloto (Harned, Korslund, Foa, \& Linehan, 2013), como en un estudio randomizado posterior en el que se compara un año de TDC vs. TDC+EP (Harned, Korslund, \& Linehan, 2014), se evidencia que al final del tratamiento, aproximadamente el $80 \%$ de los pacientes asignados a esta última intervención (TDC+PE) ya no cumple criterios para el diagnóstico de PTSD. Además, se describen resultados beneficiosos en relación a otras variables asociadas al trauma como ideación suicida, disociación y vergüenza.

La TDC ha sido adaptada también para patologías asociadas a la alimentación (i.e., bulimia nervosa y trastorno por atracón) caracteri- 
zadas por la disregulación emocional y de alta comorbilidad con el TLP. Safer desarrolló un programa grupal de 20 semanas de duración para pacientes con estos diagnósticos. En los primeros estudios, (Safer, Telch, \& Agras, 2001; Telch, Agras, \& Linehan, 2001), se compararon pacientes en TDC con pacientes en lista de espera. Se reportaron resultados favorables a la TDC, tanto en la reducción de la sintomatología como en la adherencia al tratamiento. En el 2010 se publicó un estudio comparando la TDC con otro tratamiento activo también de formato grupal (Safer, Robinson, \& Jo, 2011). La tasa de abandonos de tratamiento fue significativamente menor para el grupo de TDC $(4 \%$ vs. $33.3 \%)$ y los pacientes asignados a TDC lograron reducir la sintomatología (i.e., abstinencia de atracones y disminución de la frecuencia) más rápidamente. En otros trabajos en los que se evaluó la eficacia de la TDC completa (terapia individual, entrenamiento en habilidades, consultas telefónicas) en el tratamiento de pacientes con trastornos de la alimentación (trastorno por atracón y bulimia nerviosa) y diagnóstico de TLP comórbido se reportaron resultados prometedores (Chen, Matthews, Allen, Kuo, \& Linehan, 2008; Palmer et al., 2003).

La TDC también ha sido aplicada a pacientes con diagnóstico de trastorno depresivo mayor. En dos estudios realizados por Lynch y colaboradores (Lynch et al., 2007; Lynch, Morse, Mendelson, \& Robins, 2003) se comparó el tratamiento de TDC + medicación antidepresiva con un tratamiento únicamente farmacológico. En el primer estudio (Lynch et al., 2003) no se encontraron diferencias significativas entre ambos grupos en relación a la reducción de la sintomatología depresiva o a las tasas de remisión post-tratamiento. Sin embargo, a los 6 meses de seguimiento, el porcentaje de pacientes que continuaba en remisión fue significativamente más grande para el grupo de TDC que para el grupo control (75\% vs. $31 \%$ ). Estos resultados se confirmaron en el siguiente estudio (Lynch et al., 2007), en el que, en comparación con el grupo control, un porcentaje mayor de pacientes en la condición de TDC alcanzó criterios de remisión una vez finalizado el tratamiento.

Otra de las aplicaciones interesantes de la terapia dialéctica es la adaptación realizada por Rathus y Miller para poblaciones de adolescentes con conductas suicidas y rasgos de personalidad límite (Rathus \& Miller, 2002). La terapia dialéctico-conductual para adolescentes (TDC-A) es un tratamiento manualizado de 16 semanas de duración que incluye terapia individual y un grupo de entrenamiento en habilidades multifamiliar. Aunque el tratamiento para adolescentes sigue las directrices del tratamiento Standard en TDC, presenta también algunas modificaciones. Considerando que el ambiente familiar suele ser una fuente de conflicto para el adolescente, en la TDC-A la familia tiene un rol más activo en el tratamiento y por tanto participa del grupo de habilidades y de sesiones de terapia familiar. El tratamiento tiene una duración mas corta que el tratamiento de adultos (16 semanas vs. 1 año), pero una vez terminadas las 16 semanas el paciente tiene la opción de participar en otro grupo para "graduados" en el que se busca principalmente, la generalización y reforzamiento de habilida- 
des. En los grupos de adolescentes se enseñan los 4 módulos de habilidades (i.e. regulación emocional, mindfulness, efectividad interpersonal y tolerancia al malestar) y se agrega un quinto: el camino del medio. En este módulo se enseñan principios de validación, principios generales de modificación de conducta y habilidades para logar un comportamiento más equilibrado y menos polarizado ante ciertos dilemas dialécticos. Diversos estudios han evaluado la eficacia de la TDC-A para el tratamiento de adolescentes con rasgos de TLP, ideación o conductas suicidas y/o autolesiones (e.g., Fleischhaker et al., 2011; Mehlum et al., 2014; Rathus \& Miller, 2002). Estudios cuasi experimentales muestran que, en comparación con tratamiento habitual, la TDC- A se asocia a menos hospitalizaciones psiquiátricas durante el tratamiento, menos abandonos de terapia, y a una reducción mayor en relación a la ideación suicida, síntomas psiquiátricos generales, síntomas de TLP, (Fleischhaker et al., 2011; Mehlum et al., 2014; Rathus \& Miller, 2002). En el único RCT publicado hasta ahora (Mehlum et al., 2014) la TDC-A demostró ser superior que el tratamiento usual para la disminución de la frecuencia de autolesiones, severidad de ideación suicida y síntomas depresivos.

\section{Eficacia del entrenamiento en habilidades como componente único de tratamiento}

Desde sus primeras formulaciones, la TDC ha sido planteada como un tratamiento multicomponente, con objetivos específicos y claramente delimitados para cada uno de ellos. En congruencia con esto, la evidencia empírica acumulada hasta el momento avala la efica- cia conjunta de los distintos componentes de la TDC (i.e., terapia individual, entrenamiento en habilidades grupal y consultoría telefónica). Sin embargo, parecería ser que el entrenamiento en habilidades tendría algunas ventajas asociadas a la duración del tratamiento y el coste económico, motivando su uso como tratamiento independiente (i.e. sin terapia individual). En este formato de intervención grupal se realizan durantge 6 meses, cuatro módulos de habilidades orientados a mejorar los áreas disfuncionales claves en el paciente con TLP (i.e. problemas interpersonales, identidad, impulsividad, cognición y labilidad afectiva) mediante el entrenamiento de 4 módulos: (1) mindfulness, (2) tolerancia al dolor, (3) regulación emocional y (4) efectividad interpersonal.

El último estudio de M. Linehan publicado en JAMA (Linehan et al., 2015) señala la relevancia del EH en la TDC. En este trabajo se realizó un análisis de componentes de la terapia para determinar la eficacia aislada de cada uno de ellos y compararlos entre sí. En este ensayo clínico randomizado participaron 99 mujeres con diagnóstico de TLP y al menos dos intentos de suicidio y/o autolesiones en los últimos 5 años, y un intento de suicidio o autolesiones en las 8 semanas anteriores al comienzo del tratamiento. Las participantes fueron asignadas aleatoriamente a uno de los tres grupos: 1) TDC en formato estándar (TDC-S), 2) entrenamiento en habilidades (TDC-EH) o 3) terapia individual (TDC-I). La dosis de tratamiento se controló para que fuera similar en las tres condiciones y todos los profesionales utilizaron el protocolo de la TDC para evaluar y manejar el riesgo suicida. Las participantes asignadas a las 
tres condiciones mostraron mejorías en relación a la frecuencia y la severidad de los intentos de autoeliminación, la ideación suicida, el uso de servicios de crisis por ideación suicida y razones para vivir. En comparación con el tratamiento de terapia individual (TDC-I), las intervenciones que incluyeron habilidades (TDC-S y TDC-EH) fueron más eficaces para disminuir la frecuencia de autolesiones, los síntomas de depresión y los síntomas de ansiedad durante el periodo de tratamiento (1 año). En comparación con la TDC-I, hubo menos abandonos de terapia en la terapia estándar y los pacientes asignados a TDC-S utilizaron menos recursos de urgencias y menos hospitalizaciones psiquiátricas durante el seguimiento (1 año). Los resultados de este estudio son de gran relevancia puesto que confirman la eficacia de los tres componentes de la TDC para disminuir el número de intentos de suicidio. Además, estos hallazgos sugieren que el entrenamiento en habilidades es un componente activo y fundamental del tratamiento, ya que tanto el grupo de TDC-S como el grupo de TDC-EH fue mas eficaz que la TDC-I.

En estudios anteriores ya se había evaluado la eficacia del entrenamiento en habilidades como componente único de tratamiento (i.e. sin terapia individual). El primer estudio en esta línea fue el de Soler y colaboradores (Soler et al., 2009). En este estudio, 59 pacientes con diagnóstico de TLP fueron randomizadas a TDC-EH ( $n=29)$ o a tratamiento grupal estándar $(n=30)$. Después de 13 semanas de tratamiento, los participantes asignados a TDC-EH mostraron, en comparación con el grupo control, menores índices de depresión, ansiedad, irritabilidad, ira e inestabilidad afectiva. Además, se encontraron diferencias significativas en relación a la retención de ambos tratamientos: $34.5 \%$ de dropout en TDC-EH versus $63.4 \%$ en la intervención control. En suma, los resultados indican que la TDC-EH de 13 semanas es un tratamiento eficaz para reducir sintomatología asociada al TLP.

Si bien las habilidades fueron desarrolladas para pacientes con conductas suicidas y TLP, el carácter multimodal del entrenamiento en habilidades permite adaptarlo fácilmente a otras poblaciones. El entrenamiento en habilidades también se ha utilizado para el tratamiento de otras patologías relacionadas con la disregulación emocional. En una revisión sistemática (Valentine, Bankoff, Poulin, Reidler, \& Pantalone, 2015) se encontraron 17 trabajos en los que adaptaba el EH para distintas poblaciones como por ejemplo: trastornos de la personalidad (Long, Fulton, Dolley, \& Hollin, 2011; Soler et al., 2009), trastornos del estado de ánimo (Blackford \& Love, 2012; Feldman, Harley, Kerrigan, Jacobo, \& Fava, 2009; Harley, Sprich, Safren, Jacobo, \& Fava, 2008), trastorno por atracón (e.g., Safer \& Joyce, 2011), bulimia nerviosa (Safer et al., 2001), autolesiones no suicidas (Sambrook, Abba, \& Chadwick, 2007), trastorno oposicionista desafiante (Nelson-Gray et al., 2006) y trastorno por déficit atencional (Hirvikoski et al., 2011). De estos trabajos, en el $60 \%$ se utilizaron los 4 módulos de la TDC, mientras que en el restante $40 \%$ se omitieron uno o más módulos (e.g., los estudios en trastorno por atracón omiten el módulo de efectividad interpersonal). La comparación entre estos estudios resulta difícil debido a las diferen- 
cias metodológicas entre ellos. Los estudios se diferencian en: (a) adaptaciones específicas de las habilidades para cada población, (b) número total de sesiones de terapia (rango de 9 a 32 sesiones), (c) cantidad de horas de entrenamiento en habilidades (rango entre 17.5 y 47 horas), (d) rama comparativa (i.e. la mayoría de los estudios no comparan el EH con una intervención control), (e) finalmente, también hay grandes variaciones en cuento al porcentaje de individuos que terminan la terapia (rango entre $51 \%$ y 96\%), así como en relación al mínimo de sesiones requeridas para considerar que un individuo culminó el tratamiento. A pesar de las diferencias metodológicas entre los estudios evaluados, los autores de esta revisión concluyen -a partir de estudios en los que TDC-EH se comparó con una intervención control- que TDC-EH es eficaz como tratamiento aislado para reducir síntomas del trastorno depresivo mayor, TDAH y el trastorno por atracón (Valentine et al., 2015). De los trabajos en los que se han utilizado las habilidades como componente único de tratamiento destaca un estudio publicado por Neacsiu y colaboradores en el que se evaluó la eficacia de este entrenamiento para un grupo de sujetos con sintomatología asociada a la disregulación emocional (Neacsiu, Eberle, Kramer, Wiesmann, \& Linehan, 2014). Para este estudio se utiliza una combinación de habilidades de los 4 módulos (mindfulness, regulación emocional, efectividad interpersonal y tolerancia al malestar) para crear un programa de 16 semanas cuyo target principal es la disregulación emocional. En este estudio se utilizan habilidades para aprender a reducir la vulnerabilidad a la disre- gulación emocional, manejar crisis, aumentar el control atencional sobre los estímulos emocionales y manejar la activación fisiológica asociada a las emociones. Un total de 44 sujetos con síntomas depresivos y/o ansiosos y alta disregulación emocional (criterio de exclusión: diagnóstico de TLP), fueron randomizados a dos intervenciones grupales. Los resultados indican la superioridad de la TDC-EH en la disminución de la disregulación emocional $(\mathrm{d}=$ 1.86), el aumento de uso de habilidades ( $d=$ 1.02) y la disminución de la ansiedad ( $d=1.37$ ) pero no de la depresión $(\mathrm{d}=0.73)$. Además, la retención fue mejor en el grupo de TDC-H que en el grupo control (78\% vs. 41\%).

Considerando los estudios que hemos comentado con anterioridad, parecería ser entonces que el entrenamiento en habilidades es eficaz para el tratamiento de patologías con un alto predominio de disregulación emocional. El entrenamiento en habilidades de la TDC es un tratamiento que implica necesariamente la práctica diaria y la generalización de las habilidades en todos los contextos de relevancia para el paciente. Tomando esto en cuenta, algunos autores se han interesado en determinar la asociación entre la práctica o el uso de habilidades y la mejoría clínica. Para determinar la relación entre el uso de habilidades y el éxito terapéutico, Neacsiu y colaboradores (Neacsiu, Rizvi, \& Linehan, 2010) realizan un análisis post-hoc de resultados de un RCT previo (Linehan et al., 2006). Este artículo demuestra la importancia del uso de habilidades como mediador del éxito terapéutico, en concreto en la reducción de intentos de suicidio, de síntomas depresivos, y la mejoría en el control de 
la ira, sin embargo, es importante puntualizar que si bien este trabajo destaca la importancia del uso de habilidades en la mejoría terapéutica, estos pacientes recibían el pack completo de la terapia (i.e. terapia individual más EH), y por tanto resulta difícil aislar la eficacia de la TDC-EH. En otro estudio, Stepp y colaboradores (Stepp, Epler, Jahng, \& Trull, 2008) exploran la relación entre el uso de habilidades y la mejoría de los síntomas de TLP, concluyendo que el uso total de habilidades se relaciona con la mejoría de síntomas del trastorno (mejoría global y específica en relación a: inestabilidad afectiva, problemas de identidad y problemas en el área interpersonal). Otros estudios se han focalizado en explorar la utilidad de las distintas habilidades, reportando que no todos los módulos de habilidades se practican con igual frecuencia. Un estudio (Lindenboim, Comtois, \& Linehan, 2007) sugiere que las habilidades más practicadas por los pacientes son las habilidades de mindfulness y las de tolerancia al malestar, seguidas por las habilidades de regulación emocional y por último, las menos practicadas, las habilidades de efectividad interpersonal. Este hallazgo es interesante, ya que esta preferencia por las habilidades orientadas a la aceptación (i.e. mindfulness y tolerancia al malestar) podría indicar de algún modo una mayor utilidad. Además, encuentran que el uso de habilidades se incrementa con el tiempo, resultado que ya había sido reportado por Stepp (Stepp et al., 2008). La evidencia acumulada hasta este momento indica que los pacientes practican las habilidades (una media de 4.5 habilidades al día según el estudio de Lindenboim) y parecería ser que el uso de las habilidades se relaciona con la disminución de sintomatología asociada al TLP.

La mayoría de estudios han evaluado el paquete "original" del entrenamiento en habilidades (i.e., los 4 módulos). Aunque desde el punto de vista teórico cada uno de los módulos se focaliza en un área de disregulación específica, la evidencia aportada por algunos estudios (Lindenboim et al., 2007; Stepp et al., 2008) parecería indicar que no todas las habilidades se practican con igual frecuencia. Esta preferencia por las habilidades orientadas hacia la aceptación (i.e., mindfulness y tolerancia al malestar) podría indicar una mayor utilidad clínica. De esta forma, podría ocurrir que ante un problema interpersonal, los pacientes eligieran practicar habilidades de mindfulness orientadas a aceptar la situación o las características personales de la otra persona, en lugar de aplicar directamente habilidades de efectividad interpersonal. Para poder determinar cuales son los componentes activos del entrenamiento en habilidades, así como la especificidad terapéutica de cada módulo es necesario realizar estudios en los que se explore cada módulo independientemente de los demás. Hasta el momento, el módulo de mindfulness es el que ha recibido más atención contando con un par de estudios controlados no alegorizados (Feliu-soler et al., 2014; Soler et al., 2012). En uno de estos estudios, se comparó el módulo de mindfulness con TH en pacientes con TLP (Soler et al., 2012). Según los resultados obtenidos en un test neuropsicológico (CPT-II; Conners, 2004) el entrenamiento en mindfulness parecería asociarse a mejorías relacionadas con la atención y la impulsivi- 
dad. Concretamente, parecería afectar positivamente la capacidad de discriminar targets y no-targets y reducir errores de comisión, lo que se traduce como mayor habilidad a la hora de inhibir respuestas. Además, tiempos de reacción más largos también sugieren mejoras en la impulsividad. Estos hallazgos coinciden con los obtenidos en otras poblaciones en los que se destaca la utilidad del mindfulness sobre todo en relación a la mejoría de parámetros atencionales. Más recientemente en un estudio aleatorizado (Elices et al., in press; Soler et al., 2016), 64 pacientes con TLP fueron asignadas a un entrenamiento de 10 semanas de duración en mindfulness ( $n=32$ ) o efectividad interpersonal $(n=32)$. En comparación con el grupo de efectividad interpersonal, los pacientes que recibieron el entrenamiento en mindfulness mostraron una reducción significativa de la clínica borderline medida a través de la Borderline Symptom List-23 (Soler et al., 2013). El porcentaje de pacientes que respondieron al tratamiento según índices de significación clínica, también favoreció al grupo de mindfulness (40\% vs. $13 \%)$. Además, parecería ser que el entrenamiento en mindfulness se asocia a un aumento significativo de la capacidad de decentering, definida como la habilidad de tomar distancia de los contenidos mentales, observándolos como fenómenos de la mente (Fresco, Segal, Buis, \& Kennedy, 2007). Este resultado es interesante, ya que niveles bajos de decentering han sido asociados a distintas patologías y por tanto parece ser un indicador transdiagnósticos de psicopatología (Soler et al., 2014). En un estudio más reciente (Soler et al., 2016) en el que se comparó el módulo de mindfulness con el módulo de efectividad interpersonal, también se encontraron mejorías relativas a la impulsividad en parámetros relacionados con una mayor tolerancia a los refuerzos demorados y un aumento en la percepción del tiempo medidos mediante tests computerizados (Dougherty, Mathias, Marsh, \& Jagar, 2005). Estos resultados son interesantes para el tratamiento del TLP, ya que la baja tolerancia a las recompensas demoradas es un tipo de impulsividad que ha sido asociada a las conductas desadaptativas del trastorno (e.g., autolesiones, abuso de sustancias, atracones; Bornovalova, Lejuez, Daughters, Zachary Rosenthal, \& Lynch, 2005). En este sentido, parecería ser que el mindfulness facilitaría una postura menos reactiva y más reflexiva, permitiendo que el individuo responda a las demandas ambientales en lugar de actuar impulsivamente (Kabat-Zinn, 1990; Shapiro, Carlson, Astin, \& Freedman, 2006). En estos últimos estudios, la intervención en mindfulness se basó en la propuesta de la TDC (Linehan, 1993b), con la diferencia de que se incluyeron prácticas de meditación formal (e.g., body scan o sitting meditation). Algunas características del TLP, especialmente los altos niveles de autocrítica y de autoinvalidación, sugieren que, además de las practicas tradicionales de mindfulness y aceptación enseñadas en el contexto de la TDC, estos pacientes podrían beneficiarse de prácticas de loving kindness y compasión. Para explorar esto, Feliu-Soler y colaboradores (Feliu-Soler et al., 2015), realizaron un estudio piloto en el que se investigaron los efectos del entrenamiento en loving kindness y compasión en pacientes con TLP. Como grupo control, se utilizó el en- 
trenamiento en mindfulness estándar. Después de 3 semanas de intervención, los participantes en el grupo de loving kindness y compasión mostraron un aumento significativamente mayor que el grupo control en la aceptación del momento presente. Además, se observaron mejorías en relación a la severidad de los síntomas del TLP y en la autocrítica, así como un aumento de las capacidades de mindfulness, y de autocompasión. En forma conjunta, estos resultados indican que una intervención en loving kindness y compasión puede ser una estrategia complementaria al módulo de mindfulness de utilidad clínica para pacientes con TLP.

\section{Direcciones futuras}

Desde el primer ensayo clínico controlado publicado a principios de 1990 hasta el día de hoy, se ha acumulado evidencia suficiente para demostrar la eficacia de la TDC como tratamiento para el TLP. Aunque la cantidad de evidencia respecto a su eficacia en otros trastornos no es suficiente, parecería ser que la TDC es un tratamiento válido para distintas patologías asociadas a la disregulación emocional en general.

Los resultados del estudio de componentes de la TDC sugieren que el entrenamiento en habilidades es fundamental al menos, para el tratamiento de pacientes con TLP y conductas suicidas. Sin embargo este estudio no cuenta con la potencia estadística suficiente como para sugerir enfáticamente que el tratamiento en habilidades es tan eficaz como el tratamiento estándar.

Respecto al entrenamiento en habilidades, 20 años después de la aparición del primer manual, Linehan publica una segunda edición en la que se añaden distintos tipos de habilidades. Esta segunda edición, recoge la evidencia empírica acumulada en estos años, no solo proveniente de la TDC sino también de otras terapias basadas en la evidencia. Más estudios son necesarios para determinar qué habilidades funcionan mejor para qué tipo de pacientes o para qué tipo de problemáticas. Como plantea Linehan (Linehan \& Wilks, 2015), es de esperar que en los próximos años surjan investigaciones acerca de las nuevas habilidades y las nuevas aplicaciones de la TDC que contribuyan a mejorar y adaptar el tratamiento.

\section{Referencias}

American Psychiatric Association. (2013). Diagnostic and Statistical Manual of Mental Disorders (5th ed.). American Psychiatric Publishing.

Blackford, J. U., \& Love, R. (2012). Dialectical behaviour therapy group skills training in a communitiy mental health setting: A pilot study. International Journal of Group Psychotherapy, 61, 1-8. doi:10.1521/ ijgp.2011.61.4.645.Dialectical

Bohus, M., Dyer, A. ., Priebe, K., Krüeger, A., Kleindienst, N., Schmahl, C., ... Steil, R. (2013). Dialectical Behaviour Therapy for Post-traumatic Stress Disorder after Childhood Sexual Abuse in Patients with and without Borderline Personality Disorder: A Randomised Controlled Trial. Psychoterapy and Psychosomatics, 82, 221233. doi:10.1159/000348451

Bornovalova, M. a, Lejuez, C. W., Daughters, S. B., Zachary Rosenthal, M., \& Lynch, T. R. (2005). Impulsivity as a common process across borderline personality and substance use disorders. Clinical Psychology Review, 25, 790-812. doi:10.1016/j.cpr.2005.05.005

Carter, G. L., Willcox, C. H., Lewin, T. J., Conrad, A. M., \& Bendit, N. (2010). Hunter DBT project: randomized controlled trial of dialectical 
behaviour therapyin women with borderline personality disorder. The Australian and New Zealand Journal of Psychiatry, 44, 162-73. doi:10.3109/00048670903393621

Chen, E. Y., Matthews, L., Allen, C., Kuo, J. R., \& Linehan, M. M. (2008). Dialectical behavior therapy for clients with binge-eating disorder or bulimia nervosa and borderline personality disorder. International Journal of Eating Disorders, 41, 505-512.

Conners, B. C. K. (2004). Conners ' Continuous Performance Test II ( CPT II V . 5 ).

Crowell, S. E., Beauchaine, T. P., \& Linehan, M. M. (2009). A biosocial developmental model of borderline personality: Elaborating and extending Linehan's theory. Psychological Bulletin, 135, 495-510. doi:10.1037/ a0015616

Dougherty, D., Mathias, C. W., Marsh, D. M., \& Jagar, A. a. (2005). Laboratory behavioral measures of impulsivity. Behavior Research Methods, 37, 82-90.

Elices, M., Pascual, J.C., Portella, M.J., FeliuSoler, A., Martin-Blanco, A., Carmona, C., Soler, J. Impact of mindfulness training on borderline personality disorder: A pilot randomized trial. Under review.

Feldman, G., Harley, R., Kerrigan, M., Jacobo, M., \& Fava, M. (2009). Change in emotional processing during a dialectical behavior therapy-based skills group for major depressive disorder. Behaviour Research and Therapy, 47, 316-21. doi:10.1016/j. brat.2009.01.005

Feliu-soler, A., Pascual, J. C., Borràs, X., Portella, M. J., Martín-blanco, A., Armario, A., ... Pérez, V. (2014). Effects of Dialectical Behaviour Therapy- Mindfulness Training on Emotional Reactivity in Borderline Personality Disorder: Preliminary Results. Clinical Psychology \& Psychotherapy, 21, 363370. doi:10.1002/cpp.1837

Fleischhaker, C., Böhme, R., Sixt, B., Brück, C., Schneider, C., \& Schulz, E. (2011). Dialectical Behavioral Therapy for Adolescents (DBT-A): a clinical Trial for Patients with suicidal and self-injurious Behavior and Borderline Symptoms with a one-year Follow-up. Child and Adolescent Psychiatry and Mental Health, 5, 3. doi:10.1186/1753-2000-5-3

Foa, E. B., Riggs, D. S., Massie, E. D., \& Yarczower, M. (1995). The impact of fear activation and anger on the efficacy of exposure treatment for posttraumatic stress disorder. Behavior Therapy, 26, 487-499. doi:10.1016/S00057894(05)80096-6

Fresco, D. M., Segal, Z. V, Buis, T., \& Kennedy, S. (2007). Relationship of posttreatment decentering and cognitive reactivity to relapse in major depression. Journal of Consulting and Clinical Psychology, 75(3), 447-55. doi:10.1037/0022-006X.75.3.447

Harley, R., Sprich, S., Safren, S., Jacobo, M., \& Fava, M. (2008). Adaptation of dialectical behavior therapy skills training group for treatment-resistant depression. The Journal of Nervous and Mental Disease, 196, 136143.

Harned, M. S., Chapman, A. L., Dexter-Mazza, E. T., Murray, A., Comtois, K. a, \& Linehan, M. M. (2008). Treating co-occurring Axis I disorders in recurrently suicidal women with borderline personality disorder: a 2-year randomized trial of dialectical behavior therapy versus community treatment by experts. Journal of Consulting and Clinical Psychology, 76, 1068-1075. doi:10.1037/ a0014044

Harned, M. S., Korslund, K. E., Foa, E. B., \& Linehan, M. M. (2013). Treating PTSD in Suicidal and Self-injuring Women with Borderline Personality Disorder: Development and Preliminary Evaluation of a Dialectical Behavior Therapy Prolonged Exposure Protocol. Behaviour Research and Therapy, 50, 381-386. doi:10.1016/j. brat.2012.02.011.Treating

Harned, M. S., Korslund, K. E., \& Linehan, M. M. (2014). A pilot randomized controlled trial of Dialectical Behavior Therapy with and without the Dialectical Behavior Therapy Prolonged Exposure protocol for suicidal and self-injuring women with borderline personality disorder and PTSD. Behaviour Research and Therapy, 55C, 7-17. doi:10.1016/j.brat.2014.01.008 
Hirvikoski, T., Waaler, E., Alfredsson, J., Pihlgren, C., Holmström, A., Johnson, A., ... Nordström, A. L. (2011). Reduced ADHD symptoms in adults with ADHD after structured skills training group: Results from a randomized controlled trial. Behaviour Research and Therapy, 49, 175-185. doi:10.1016/j. brat.2011.01.001

Kabat-Zinn, J. (1990). Full catastrophe living: Using the wisdom of your body and mind to face stress, pain, and illness. New York, NY: Delacorte.

Koons, C. R., Robins, C. J., Tweed, J. L., Lynch, T. R., Gonzalez, A. a., Morse, J. Q., ... Bastian, L. a. (2001). Efficacy of dialectical behavior therapy in women veterans with borderline personality disorder. Behavior Therapy, 32, 371-390. doi:10.1016/S00057894(01)80009-5

Lindenboim, N., Comtois, K. A., \& Linehan, M. M. (2007). Skills Practice in Dialectical Behavior Therapy for Suicidal Women Meeting Criteria for Borderline Personality Disorder. Cognitive and Behavioral Practice, 14, 147156. doi:10.1016/j.cbpra.2006.10.004

Linehan, M. M. (1993). Cognitive-Behavioral Treatment of Borderline Personality Disorder. New York, NY: The Guilford Press.

Linehan, M. M. (1993). Skills training manual for treating borderline personality disorder. Guilford Press.

Linehan, M. M., Armstrong, H. E., Suarez, A., Allmon, D., \& Heard, H. L. (1991). CognitiveBehavioral Treatment of Chronically Parasuicidal Borderline Patients. Archives of General Psychiatry, 48, 1060-1064.

Linehan, M. M., Comtois, K. A., Murray, A. M., Brown, M. Z., Gallop, R. J., Heard, H. L., ... Lindenboim, N. (2006). Two-year randomized controlled trial and follow-up of dialectical behavior therapy vs therapy by experts for suicidal behaviors and borderline personality disorder. Archives of General Psychiatry, 63, 757-66. doi:10.1001/ archpsyc.63.7.757

Linehan, M. M., Dimeff, L. a., Reynolds, S. K., Comtois, K. A., Welch, S. S., Heagerty, P., \& Kivlahan, D. R. (2002). Dialectical behavior therapy versus Comprehensive Validation
Therapy plus 12-step for the treatment of opioid dependent women meeting criteria for borderline personality disorder. Drug and Alcohol Dependence, 67, 13-26. doi:10.1016/S0376-8716(02)00011-X

Linehan, M. M., Korslund, K. E., Harned, M. S., Gallop, R. J., Lungu, A., Neacsiu, A. D., ... Murray-gregory, A. M. (2015). Dialectical Behavior Therapy for High Suicide Risk in Individuals With Borderline Personality Disorder A Randomized Clinical Trial and Component Analysis. JAMA, 98195, 1-8. doi:10.1001/jamapsychiatry.2014.3039

Linehan, M. M., Schmidt, H., Dimeff, L. A., \& Comtois, K. A. (1999). Dialectical Behavior Therapy for patients with borderline personality disorder and drug-dependence. The American Journal of Addictions, 8, 279292.

Linehan, M. M., Tutek, D. a., Heard, H. L., \& Armstrong, H. E. (1994). Interpersonal outcome of cognitive behavioral treatment for chronically suicidal borderline patients. American Journal of Psychiatry, 151, 17711776.

Linehan, M. M., \& Wilks, C. (2015). The Course and Evolution of Dialectical Behavior. American Journal of Psychotherapy, 69, 97110.

Long, C. G., Fulton, B., Dolley, O., \& Hollin, C. R. (2011). Dealing with Feelings: the effectiveness of cognitive behavioural group treatment for women in secure settings. Behavioural and Cognitive Psychotherapy, 39, 243-247. doi:10.1017/ S1352465810000573

Lynch, T. R., Cheavens, J. S., Cukrowicz, K. C., Thorp, S. R., Bronner, L., \& Beyer, J. (2007). Treatment of older adults with co-morbid personality disorder and depression: a dialectical behavior therapy approach. International Journal of Geriatric Psychiatry, 22, 131-143. doi:10.1002/gps

Lynch, T. R., Morse, J. Q., Mendelson, T., \& Robins, C. J. (2003). Dialectical behavior therapy for depressed older adults: a randomized pilot study. The American Journal of Geriatric Psychiatry: Official Journal of the American Association for Geriatric Psychiatry, 11, 33-45. 
McMain, S. F., Links, P. S., Gnam, W. H., Guimond, T., Cardish, R. J., Korman, L., \& Streiner, D. L. (2009). A randomized trial of dialectical behavior therapy versus general psychiatric management for borderline personality disorder. The American Journal of Psychiatry, 166, 1365-1374. doi:10.1176/ appi.ajp.2009.09010039

Mehlum, L., Tørmoen, A. J., Ramberg, M., Haga, E., Diep, L. M., Laberg, S., ... Grøholt, B. (2014). Dialectical behavior therapy for adolescents with repeated suicidal and self-harming behavior: a randomized trial. Journal of the American Academy of Child and Adolescent Psychiatry, 53, 1082-1091. doi:10.1016/j.jaac.2014.07.003

Neacsiu, A. D., Eberle, J. W., Kramer, R., Wiesmann, T., \& Linehan, M. M. (2014). Dialectical behavior therapy skills for transdiagnostic emotion dysregulation: a pilot randomized controlled trial. Behaviour Research and Therapy, 59, 40-51. doi:10.1016/j.brat.2014.05.005

Neacsiu, A. D., Rizvi, S. L., \& Linehan, M. M. (2010). Dialectical Behavior Therapy Skills Use as a Mediator and Outcome of Treatment for Borderline Personality Disorder. Behavior Research and Therapy, 48, 832-839. doi:10.1016/j.brat.2010.05.017. Dialectical

Nelson-Gray, R. O., Keane, S. P., Hurst, R. M., Mitchell, J. T., Warburton, J. B., Chok, J. T., \& Cobb, A. R. (2006). A modified DBT skills training program for oppositional defiant adolescents: promising preliminary findings. Behaviour Research and Therapy, 44, 1811-1820. doi:10.1016/j.brat.2006.01.004

Palmer, R. L., Birchall, H., Damani, S., Gatward, N., McGrain, L., \& Parker, L. (2003). A dialectical behavior therapy program for people with an eating disorder and borderline personality disorder-description and outcome. International Journal of Eating Disorders, 33, 281-286.

Pasieczny, N., \& Connor, J. (2011). The effectiveness of dialectical behaviour therapy in routine public mental health settings: An Australian controlled trial.
Behaviour Research and Therapy, 49, 4-10. doi:10.1016/j.brat.2010.09.006

Rathus, J. H., \& Miller, A. L. (2002). Dialectical behavior therapy adapted for suicidal adolescents. Suicide and Life-Threatening Behavior, 32, 146-157.

Safer, D. L., \& Joyce, E. E. (2011). Does rapid response to two group psychotherapies for binge eating disorder predict abstinence? Behaviour Research and Therapy, 49, 339-45. doi:10.1016/j.brat.2011.03.001

Safer, D. L., Robinson, A. H., \& Jo, B. (2011). Outcome from a randomized controlled trial of group therapy for binge eating disorder: Comparing Dialectical Behavior Therapy adapted to binge eating to an active comparison group therapy. Behavior Therapy, 41, 106-120. doi:10.1016/j. beth.2009.01.006.Outcome

Safer, D. L., Telch, C. F., \& Agras, W. S. (2001). Dialectical behavior therapy for bulimia nervosa. The American Journal of Psychiatry, 158, 632-634. Recuperado de http://www. ncbi.nlm.nih.gov/pubmed/18348281

Sambrook, S., Abba, N., \& Chadwick, P. (2007). Evaluation of DBT emotional coping skills groups for people with parasuicidal behaviours. Behavioural and Cognitive Psychotherapy, 35, 241-244.

Shapiro, S. ., Carlson, L. E., Astin, J. ., \& Freedman, B. (2006). Mechanisms of mindfulness. Journal of Clinical Psychology, 62, 373-386.

Soler, J., Elices, M., Pascual, J.C, Martin-Blanco, A., Feliu-Soler, A., Carmona, C., Portella, M.J. Effects of mindfulness training on different components of impulsivity in borderline personality disorder. Under review.

Soler, J., Franquesa, A., Feliu-Soler, A., Cebolla, A., Garcia-Campayo, J., Tejedor, R., ... Portella, M. J. (2014). Assesing decentering: Validation, psychometric properities and clinical usefulness of the Experiences Questionnaire in a Spanish sample. Behavior Therapy. doi:http://dx.doi.org/10.1016/j. beth.2014.05.004

Soler, J., Pascual, J. C., Tiana, T., Cebrià, A., Barrachina, J., Campins, M. J.,... Pérez, V. (2009). Dialectical behaviour therapy skills 
training compared to standard group therapy in borderline personality disorder: a 3-month randomised controlled clinical trial. Behaviour Research and Therapy, 47, 353-358. doi:10.1016/j.brat.2009.01.013

Soler, J., Valdepérez, A., Feliu-Soler, A., Pascual, J. C., Portella, M. J., Martín-Blanco, A.,... Pérez, V. (2012). Effects of the dialectical behavioral therapy-mindfulness module on attention in patients with borderline personality disorder. Behaviour Research and Therapy, 50, 150-7. doi:10.1016/j. brat.2011.12.002

Soler, J., Vega, D., Feliu-Soler, A., Trujols, J., Soto, A., Elices, M.,... Pascual, J. C. (2013). Validation of the Spanish version of the Borderline Symptom List, short form (BSL-23). BMC Psychiatry, 13, 139. doi:10.1186/1471-244X-13-139

Stepp, S. D., Epler, A. J., Jahng, S., \& Trull, T. J. (2008). The effect of dialectical behavior therapy skills use on borderline personality disorder features. Journal of Personality Disorders, 22, 549-63. doi:10.1521/ pedi.2008.22.6.549

Stoffers, J., Völlm, B. A., Rücker, G., Timmer, A., Huband, N., \& Lieb, K. (2012). Psychological therapies for people with borderline personality disorder (Review). En Cochrane Database of Systematic Reviews (Cochrane D.). John Wiley \& Sons. doi:10.1002/14651858.CD005652

Telch, C. F., Agras, W. S., \& Linehan, M. M. (2001). Dialectical behavior therapy for binge eating disorder. Journal of Consulting and Clinical Psychology, 69, 1061-1065. doi:10.1037/0022-006X.69 6.1061

Turner, R. . (2000). Naturalistic evaluation of dialectical behavior therapy-oriented treatment for borderline personality disorder. Cognitive and Behavioral Practice, 7, 413-419.

Valentine, S. E., Bankoff, S. M., Poulin, R. M., Reidler, E. B., \& Pantalone, D. W. (2015). The Use of Dialectical Behavior Therapy Skills Training as Stand-Alone Treatment: A Systematic Review of the Treatment Outcome Literature. Journal of Clinical Psychology, 71, 1-20. doi:10.1002/jclp.22114
Verheul, R., Van Den Bosch, L. M. C., Koeter, M. W. J., De Ridder, M. a J., Stijnen, T., \& Van Den Brink, W. (2003). Dialectical behaviour therapy for women with borderline personality disorder: 12-month, randomised clinical trial in The Netherlands. The British Journal of Psychiatry: The Journal of Mental Science, 182, 135-140.

\section{Agradecimientos}

JS es miembro de PROMOSAM: Investigación en procesos, mecanismos y tratamientos psicológicos para la promoción de la salud mental, (Red de Excelencia PSI2014-56303REDT) fondos del Ministerio de Economía y Competitividad (2014). 
